\title{
Surface Undulations in Explosive Crystallization: A Thermal Instability
}

\author{
Wim van Saarloos and John D. Weeks \\ Bell Laboratories, Murray Hill, New Jersey 07974
}

(Received 19 May 1983)

\begin{abstract}
It is argued that surface undulations observed after self-sustained rapid crystallization of amorphous films result from a thermal instability that induces a periodically varying crystallization rate. Its physical origin is discussed for a simple nonlinear heat-conduction model which yields good agreement with experimental observations. A numerical analysis of the nonlinear oscillations shows that these may, in turn, bifurcate via a series of period-doubling bifurcations.
\end{abstract}

PACS numbers: $61.50 . \mathrm{Cj}, 64.70 . \mathrm{Kb}, 68.55 .+\mathrm{b}$

Recently there has been much interest in instabilities occurring during crystal growth, particularly those leading to dendritic growth and the morphological instabilities of the crystal-melt interface. ${ }^{1}$ Here we analyze a much simpler thermal instability which can explain phenomena observed during the rapid crystallization of metastable amorphous films when initiated by a laser pulse or by impact with a stylus.

Under appropriate experimental conditions, layers of amorphous $\mathrm{Sb}^{2,3} \mathrm{Ge},{ }^{4-7} \mathrm{Si}^{8}$ and other materials ${ }^{9}$ can "explosively" crystallize, the speed of the crystallization front being of the order of meters per second. Since crystallization rates are strongly dependent on temperature, it was realized long ago ${ }^{2}$ that this phenomenon is associated with the temperature rise induced by the latent heat liberated in the amorphous to crystalline $(a-c)$ transition. If the latent heat liberated is too small or the heat loss too great, the crystallization wave cannot sustain itself and dies out. One then has to initiate the process repeatedly by scanning the layer with a laser beam. $5,6,8,10$

In materials like $\mathrm{Sb},{ }^{2,3} \mathrm{Bi},{ }^{9}$ or $\mathrm{Yb},{ }^{9}$ on the other hand, self-sustained crystallization waves have been observed that propagate through the whole layer after being initiated at a single spot. In several of the latter experiments the crystalline phase exhibits periodic variations in the height of the layer..$^{2,3}$ Far from the initiation point, the structure is essentially one dimensional, resembling a frozen-in pattern of parallel water waves near a beach. The experiments of Wickersham, Bajor, and Greene ${ }^{3}$ suggest that these undulations are induced by a thermal instability. Here, we argue that they indeed result from an instability of a steadily advancing $a-c$ front to one with an oscillating growth rate.

In view of the experimental observations, we analyze the propagation of a straight front, ne- glecting temperature differences in the vertical and lateral directions. In the laboratory frame, the propagation is taken to be in the positive $x^{\prime}$ direction; it is more convenient, however, to use the coordinate frame $x=x^{\prime}-\int^{t} d t^{\prime} V\left(t^{\prime}\right)$ comoving with the front so that the $a$ phase is at $x>0$ and the $c$ phase at $x<0$. Here $V$ is the interface velocity or growth rate. Using this frame, we follow Gilmer and Leamy ${ }^{11}$ and assume a balance equation of the form ${ }^{12}$

$$
\frac{\partial T}{\partial t}=\kappa \frac{\partial^{2} T}{\partial x^{2}}+V \frac{\partial T}{\partial x}-\Gamma\left(T-T^{0}\right)+q V \delta(x) .
$$

The first term on the right hand side gives the heat conduction through the layer ( $\kappa$ is the thermal diffusivity, assumed for simplicity equal in the $a$ and $c$ phases); the second term results from the transformation to the moving frame; the third one crudely describes the heat loss to the substrate at temperature $T^{0}$. The fourth contribution is the source term due to the latent heat $L V$ liberated per unit of time and area at the $a-c$ boundary (if $C$ is the specific heat per unit volume, $q=L /$ $C)$. The boundary condition for $T$ is $T(x, t) \rightarrow T^{0}$ for $x \rightarrow \pm \infty$.

Equation (1) becomes nonlinear through the dependence of $V$ on the temperature. Following standard practice, ${ }^{13}$ we assume that $V$ is an explicit function only of the $a-c$ boundary temperature $T^{b}(t)=T(x=0, t)$,

$$
V=V\left(T^{b}(t)\right) \text {. }
$$

For concreteness, we consider a dependence of $V$ on $T^{b}$ as observed for crystal growth from a melt $^{13}$ (Fig. 1). The $a-c$ growth rate is thought to be of a simjlar form, but precise data are only available on the low-temperature side where $V$ $\propto \exp (-Q / k T) .^{14}$ It is precisely on this side where the thermal instability discussed below occurs. 


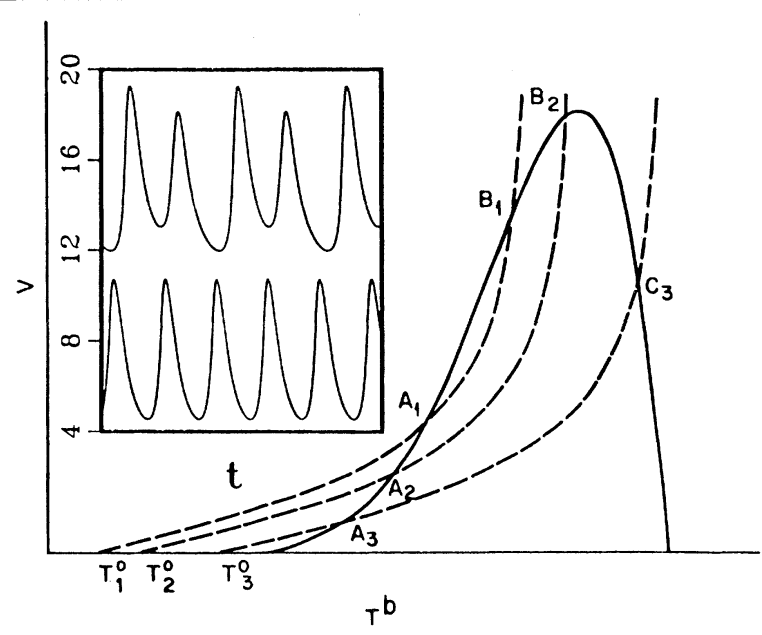

FIG. 1. Solid line: a typical curve for the $a-c$ growth velocity as a function of the $a-c$ boundary temperature. The dashed lines give the relationship between $V_{\mathrm{ss}}$ and $T_{\mathrm{ss}}{ }^{b}$ for which Eq. (1) has steady-state solutions. Inset: $V$ as a function of time for two different values of $T^{0}$ which produce oscillatory growth rates. The upper curve (obtained by adding 8 units to $V$ ) is a "period 2" solution.

In steady-state propagation, $T^{b}=T_{\mathrm{ss}}{ }^{b}$ is constant, and one can analyze Eq. (1) for a fixed velocity $\boldsymbol{V}_{\mathrm{ss}}$. Then Eq. (1) is linear and is solved by an exponential profile of the form $T=T_{\mathrm{ss}}{ }^{b} \exp (-a x)$ for $x>0$ and similarly for $x<0$, provided that $V_{\mathrm{ss}}$ and $T_{\mathrm{ss}}{ }^{b}$ are related by ${ }^{11}$

$$
V_{\mathrm{ss}}=2(\kappa \Gamma)^{1 / 2}\left[\left(q / \Delta T_{\mathrm{ss}}{ }^{b}\right)^{2}-1\right]^{-1 / 2} \text {. }
$$

Here $\Delta T_{\mathrm{ss}}{ }^{b}=T_{\mathrm{ss}}{ }^{b}-T^{0}$ is the temperature rise at the boundary. This function is drawn in Fig. 1 with a dashed line for several values of $T^{0}$, keeping $\kappa$ and $\Gamma$ fixed.

Not all hypothetical $V_{\text {ss }}$ satisfying Eq. (3) are consistent with the physically possible growth rates given in Eq. (2), so that the actual steadystate velocity and boundary temperature are determined by the intersection of these curves, as shown in Fig. 1. As in the experiments, ${ }^{3}$ different steady-state values are obtained by changing $T^{0}$.

To discuss the stability of these possible steadystate solutions, we have to consider the effect of a perturbation on the heat flow and heat loss as well as on the amount of heat generated. We dis tinguish between three different types of intersections, labeled $A, B$, and $C$ in Fig. 1. The most interesting behavior occurs for points $B$. Consider the steady state at $B_{2}$ where the slope of the growth rate curve is small but positive. Here a

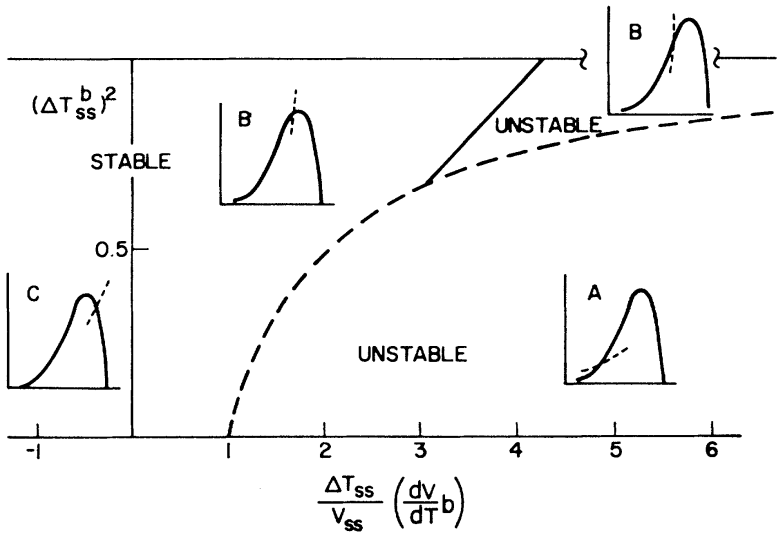

FIG. 2. Stability diagram for the various steady-state solutions whose locations on the growth-rate curve are indicated schematically. The (dimensionless) slope of the function (2) at the steady-state point is plotted along the horizontal axis and $\left(\Delta T_{\mathrm{ss}}{ }^{b}\right)^{2}$ (in units of $q^{2}$ ) along the vertical axis. The dashed line separates the points denoted by $A$ and $B$ in Fig. 1. The steady-state points $B$ to the right of the solid line are unstable. However, the full nonlinear equation can admit stable periodic solutions in this region, as illustrated in Fig. 1.

large increase in $T^{b}$, and so a relatively large increase in heat loss and heat flow away from the boundary, is associated with only a small increase in velocity (and so heat generation). We therefore expect this point to be stable. For intersections with a large slope, like $B_{1}$, the situation is reversed and these points should therefore be unstable, provided that $\Gamma$ is not too large. Along similar lines, one concludes that intersections at $A$ must be unstable and those at $C$ stable.

These considerations are borne out by a straightforward linear stability analysis of the steadystate solutions, using the formal solution to Eq. (1), valid after times long enough to neglect the initial conditions:

$$
\Delta T^{b}(t)=q \int_{-\infty}^{0} d x(4 \pi \kappa \tau)^{-1 / 2} \exp \left(-\Gamma \tau-x^{2} / 4 \kappa \tau\right)
$$

where $\tau=\tau(x, t)$ is implicitly given by

$$
x=-\int_{t-\tau}^{t} d t^{\prime} V\left(T^{b}\left(t^{\prime}\right)\right) .
$$

The resulting stability diagram is drawn in Fig. 2. In agreement with the physical arguments given above, steady-state points $C$ are stable and those of type $A$ unstable. The ones of type $B$ are unstable in the case $\left(\Delta T_{\mathrm{ss}}{ }^{b} / q\right)^{2}>2 / 3$ (or $\Gamma<V_{\mathrm{ss}}{ }^{2}$ ) $8 \kappa)$ when they lie to the right of the solid line, so that the dimensionless slope (plotted along the 
horizontal axis) reaches some value between 3 and $2+\sqrt{5} \approx 4.236$. This is an oscillatory instability: For values of the slope larger than the critical value, the boundary temperature and speed of the front oscillate periodically in time (see inset, Fig. 1). Successive period-doubling bifurcations will be discussed later.

Having understood the stability, it remains to understand the physical origin of the oscillations. In the steady state, when Eq. (5) reduces to $|x|$ $=V_{\mathrm{ss}} \tau$, the main contribution to the integral (4) comes from those positions $x$ for which $\Gamma \tau+x^{2} /$ $4 \kappa \tau \leqslant 2$, or $\tau \leqslant 2 /\left(\Gamma+V_{\mathrm{ss}}^{2} / 4 \kappa\right)=\tau_{m}$. Thus, contributions to $T^{b}$ from the heat released at positions further back than $d_{m}=V_{s s} \tau_{m}$ are essentially negligible; $\tau_{m}$ sets the time scale over which changes in $T^{b}$ take place and becomes shorter the larger the velocity is. To understand the oscillatory solutions, consider a small perturbation with an increased velocity around a steady-state solution in the case of small damping $\left(\Gamma \ll V_{\mathrm{ss}}^{2} / 4 \kappa\right)$. A small decrease in $\tau$ makes a Gaussian $\tau^{1 / 2}$ $\times \exp \left(-y^{2} / 4 \tau\right)$ narrower: The center increases whereas the wings at $|y|>(2 \tau)^{1 / 2}$ decrease. For the (slightly damped) Gaussians in the integrand of Eq. (4) with $|x|<2 \kappa / V_{\mathrm{ss}}=d_{m} / 4$ the perturbation therefore results in an increase of $T^{b}$, while it decreases the contributions of those at distances further away. ${ }^{15}$ Thus, when the growth rate speeds up, the boundary can move ahead so rapidly that the heat diffusion from positions not immediately behind the boundary is not able to keep up. Consequently, $T^{b}$ and the front velocity drop, after which more heat diffuses towards the boundary, and the velocity can rise again.

There is evidence ${ }^{3}$ that the surface undulations are accompanied by variations in grain size and in the extent of completeness of the $a-c$ transformation (there are considerable density differences between the $a$ and $c$ phases). It is quite natural to expect that the predicted oscillations in $V$ and $T^{b}$ could give rise to such variations in the crystallization process, and hence induce the surface undulations. Moreover, our predictions agree well with the following observations of Wickersham, Bajor, and Greene ${ }^{3}$ : (i) Since the time scale is set by $\tau_{m}$, we expect the period of the oscillations to be of this order. The linear stability analysis confirms this, and in the experimentally relevant case where $\Gamma \ll V_{\mathrm{av}}^{2} / 4 \kappa$, we find for the wavelength $\lambda$ of the undulations $\lambda \approx \tau_{m} V_{\mathrm{av}} \approx 8 \kappa / V_{\mathrm{av}}$. Here $V_{\text {av }}$ is the average velocity. This result is in good agreement with the experimental values measured. ${ }^{16}$ (ii) Let $T^{*}$ be the substrate temper- ature above which self-sustained explosive crystallization is possible. For $T^{0}$ slightly above $\boldsymbol{T}^{*}$, say $T^{0}=T_{1}{ }^{0}$, as shown in Fig. 1 , the slope at $B_{1}$ is steep and the growth rate will oscillate. If $T^{0}$ increases to $T_{2}{ }^{0}$, say, the point $B_{2}$ becomes stable. Thus, in our model, the oscillations occur for $T^{0}$ slightly above $T^{*}$ and cease at higher temperatures, much like the experimental observation that the "surface roughness was found to decrease as the triggering temperature was increased above $T^{*} ., 3$

A numerical analysis of the nonlinear periodic oscillations given by Eqs. (1) and (2) shows that these, in turn, bifurcate via a sequence of period-doubling bifurcations ${ }^{17}$ when the substrate temperature is lowered. One solution with a period twice the basic period $\tau_{m}$ is shown in the inset of Fig. 1. Such solutions occur if the velocity comes close to the maximum of the growth-rate curve (2). These Feigenbaum sequences ${ }^{17}$ have been analyzed mostly in models with a finite-dimensional phase space. The model studied here for the "infinite-dimensional" temperature field is simple enough to permit a detailed numerical analysis. Furthermore, in the region in Fig. 2 where the solid and dashed lines cross, the nonlinear behavior of the model can be studied analytically by making use of the theory of codimension two bifurcations. These results will be discussed in a future publication.

We are grateful to G. H. Gilmer, P. C. Hohenberg, K. A. Jackson, and D. S. Fisher for helpful discussions.

\footnotetext{
${ }^{1}$ For a general review see J. S. Langer, Rev. Mod. Phys. 52, 1 (1980).

${ }^{2}$ C. C. Coffin and S. Johnston, Proc. Roy. Soc. London, Ser. A 146, 564 (1934).

${ }^{3}$ C. E. Wickersham, G. Bajor, and J. E. Greene, Solid State Commun. 27, 17 (1978); C. E. Wickersham, Ph.D. thesis, University of Illinois, 1978 (unpublished).

${ }^{4}$ T. Takamori, R. Messier, and R。Roy, Appl. Phys. Lett. 20, 201 (1972); A. Mineo, A. Matsuda, T. Kurosu, and M. Kikuchi, Solid State Commun. 13, 329 (1973); C. E. Wickersham, Solid State Commun. 34, 907 (1980).

${ }^{5}$ R. B. Gold, J. F. Gibbons, T. J. Magee, J. Peng, R. Ormond, V. R. Deline, and C. A. Evans, in Laser and Electron Beam Processing of Materials, edited by C. W. White and P. S. Peercy (Academic, New York, 1980), p. 221.

${ }^{6}$ H. J. Zeiger, J. C. C. Fan, B. J. Palm, R. P. Gale, and R. L. Chapman, Ref. 5, p. 234, and Phys. Rev. B 25, 4002 (1982), and Appl. Phys. Lett. 6ㅜ, 158 (1980), and $37,292(1980)$.

${ }^{7}$ H. J. Leamy, W. L. Brown, G. K. Celler, G. Foti,
} 
G. H. Gilmer, and J. C. C. Fan, Appl. Phys. Lett. 38, 137 (1981); R. Koba and C. E.Wickersham, Appl. Phys. Lett. 40, 672 (1982)。

${ }^{8}$ G. Auvert, D. Bensahel, A. Georges, V. T. Nguyen, P. Henoc, F. Morin, and P. Coissard, Appl. Phys. Lett. 38, 613 (1981), and 39, 724 (1981); R. A. Lemons and M. A. Bosch, Appl. Phys. Lett. 39, 343 (1981).

${ }^{9}$ S. V. Krishnaswamy, R. Messier, P. Swab, L. L. Tongson, and K. Vedam, J. Electron. Mater. 10, 433 (1981); V. M. Kuz'menko and V. I. Mel'nikov, Zh. Eksp. Teor. Fiz. $\underline{82}, 802$ (1982) [ Sov. Phys. JETP $\underline{55}, 474$ (1982)].

${ }^{10}$ Zeiger et al. (Ref. 6) have analyzed this case in some detail. However, they make the physically questionable approximation that the temperature at the boundray remains constant despite possible variations in the interface velocity. This approximation is inconsistent with the mechanism leading to propagating oscillatory crystallization waves discussed here. Furthermore, as noted by the authors, it causes numerical convergence problems and can induce unphysical negative interface velocities at low substrate temperatures.

${ }^{11}$ G. H. Gilmer and H. J. Leamy, Ref. 5, p. 227.

${ }^{12} \mathrm{In}$ Ge there is probably a liquid layer in between the $a$ and $c$ phases (Refs. 7 and 11). Whether such a zone is present in Sb is not clear, but it is certainly absent in $\mathrm{Yb}$ and $\mathrm{Bi}$ (Ref. 9). Though we do not take a liquid zone into account, we believe that its presence would not modify the thermal instability qualitatively, since its thickness $(\approx 0.05 \mu \mathrm{m})$ is much smaller than the wavelength of the undulations $(\approx 1 \mu \mathrm{m})$.

${ }^{13}$ See, e.g., R. F. Sekerka, in Proceedings of the International School of Crystallography, Erice, 1981, Seventh Course (Reidel, Dordrecht, 1982), p. 489.

${ }^{14}$ G. L. Olson, S. A. Kokorowski, J. A. Roth, and L. D. Hess, in Laser-Solid Interactions, edited by J. Narayan, W. L. Brown, and R. A. Lemons (NorthHolland, Amsterdam, 1983).

${ }^{15}$ In the opposite case $\left(\Gamma>V_{S S}{ }^{2} / 4 \kappa\right)$ all damped Gaussians in Eq. (4) respond similarly to a perturbation and all disturbances decay monotonically.

${ }^{16}$ We estimate $\kappa \approx(1.6 \pm 0.4) \times 10^{-6} \mathrm{~m}^{2} / \mathrm{s}$ for the $c$ phase in Ref. 3. Since the measured $V_{\mathrm{av}}$ ranges from 2 to 5 $\mathrm{m} / \mathrm{s}$, we predict $\lambda \approx 2-6 \mu \mathrm{m}$. Measured values are of this order, with $\lambda \approx 1.5 \mu \mathrm{m}$ for the case shown in Fig. 1 of Ref. 3.

${ }^{17}$ M. J. Feigenbaum, J. Stat. Phys. 19, 25 (1978), and 21, 669 (1979). 